\title{
Burnout docente: un análisis bibliométrico sobre la producción científica indexada en Scopus
}

\section{Teacher burnout: a bibliometric analysis of scientific production indexed on Scopus}

\author{
Mariel Cecilia-Martín. \\ Universidad Nacional de Mar del Plata (Argentina). \\ mariel@mdp.edu.ar \\ Laura Rubio-González. \\ Universidad Pablo de Olavide. \\ Irubgon@upo.es \\ Juan-Agustín Morón-Marchena. \\ Universidad Pablo de Olavide. \\ jamormar1@upo.es \\ David Cobos-Sanchiz. \\ Universidad Pablo de Olavide. \\ dcobos@upo.es
}

\section{RESUMEN.}

Introducción: Las modificaciones técnicas y organizativas del ámbito laboral ocasionan la aparición de riesgos psicosociales emergentes. La profesión docente constituye una profesión de riesgo en la que se refieren riesgos psicosociales, singularmente el Burnout. Objetivos: Conocer la productividad diacrónica de los veinte últimos años sobre Burnout y Burnout en la Enseñanza. Verificar si se cumple la Ley de Price. Determinar qué países son los que producen más documentos científicos sobre el tema abordado. Señalar cuáles son las áreas temáticas principales y las revistas dominantes en las que se enmarcan las publicaciones. Establecer las conexiones entre los distintos descriptores relacionados con las palabras clave analizadas. Métodos: Se ha desarrollado un estudio bibliométrico descriptivo. La muestra estuvo constituida por documentos indexados en la base de datos Scopus. Las unidades de análisis fueron las palabras clave, extraídas de forma automática. Con las mismas se crearon mapas bibliométricos aplicando una metodología basada en análisis de co-palabras, técnicas de agrupamiento y técnicas de visualización. Resultados: Se ha constatado un aumento exponencial de la producción en este tópico de investigación, confirmándose la Ley de Price. Solo diez países acaparan casi el $70 \%$ del total de la producción. Medicina, Enfermería, Psicología y Ciencias Sociales son las áreas principales en la investigación sobre la temática. Conclusiones: Se constata que la temática analizada es un frente de investigación multidisciplinar de plena actualidad y que la producción científica se encuentra altamente concentrada. Se ha constatado que los mapas bibliométricos constituyen un instrumento adecuado para comprender la estructura temática de este tópico de investigación.

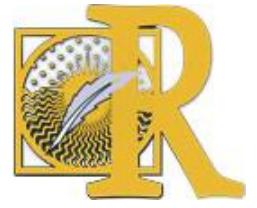

Fecha de recepción: 05-06-2020 Fecha de aceptación: 09-06-2020 


\title{
PALABRAS CLAVE.
}

Burnout; enseñanza; bibliometría; producción científica, salud laboral.

\begin{abstract}
.
Introduction: The technical and organizational changes in the work environment lead to the appearance of emerging psychosocial risks. The teaching profession constitutes a risk profession when it comes to psychosocial risks, in particular Burnout. Objectives: To learn about the diachronic productivity of the last twenty years on Burnout and Burnout in Teaching. To verify if Price's Law is fulfilled. To determine which countries, produce the most scientific documents on the subject. To indicate what the main thematic areas are and the dominant journals into which the publications are classified. To establish the connections between the different descriptors with regard to the keywords analysed. Methods: A descriptive bibliometric analysis has been carried out. The sample consisted of documents indexed in the Scopus database. The units of analysis were the keywords that were extracted automatically. Bibliometric maps were created using a methodology based on co-word analysis, clustering techniques and visualization techniques. Results: An exponential increase in production has been observed in this research topic, confirming Price's Law. Only ten countries account for almost $70 \%$ of total production. Medicine, Nursing, Psychology and Social Sciences are the main areas of research on the topic. Conclusions: It has been verified that the topic under analysis is a current multidisciplinary research front and that scientific production is highly concentrated. It has been verified that bibliometric maps are an adequate instrument to understand the thematic structure of this research topic.
\end{abstract}

\section{KEY WORDS.}

Burnout; teaching; bibliometrics; scientific production; Occupational health.

\section{Introducción.}

Los riesgos psicosociales son aspectos del diseño, la organización, dirección del trabajo y sus contextos sociales y organizacionales, que tienen el potencial de causar un daño psicológico, físico o social al individuo (Gil-Monte, 2014). La Agencia Europea para la Seguridad y la Salud en el Trabajo ya advirtió hace más de una década que las modificaciones técnicas y organizativas que se están produciendo en el ámbito laboral, junto a los cambios sociodemográficos y políticos, incluyendo la globalización, están ocasionando la aparición de riesgos psicosociales emergentes que pueden producir un mayor estrés laboral y, en consecuencia, repercutir sobre la salud y seguridad de los trabajadores (European Agency for Safety and Health at Work, 2007). El estrés se entiende como un proceso de índole psicosocial en el que no sólo ha de tenerse en cuenta la respuesta fisiológica del organismo, sino principalmente, las características del ambiente social y factores personales (Vallejo, Aja y Plaza, 2018).

En concreto, el estrés laboral puede ser considerado como un estado psicológico, caracterizado por reacciones emocionales, cognitivas, fisiológicas y del comportamiento. También, es considerado una reacción o respuesta física, emocional, cognitiva y psicológica ante situaciones laborales demandantes que exceden los recursos laborales y personales, y

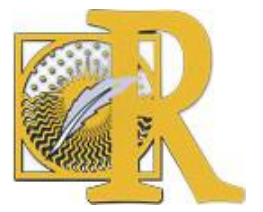


sobre las cuales no se tiene control para hacerles frente, lo cual genera consecuencias en la salud de los trabajadores (Patlán, 2019).

Se trata de un problema que forma parte de la vida diaria de una gran parte de la población mundial porque, en las últimas décadas, el número de casos sigue creciendo hasta el punto que ha empezado a ser considerado un problema de salud pública (Lima y Lima-Filho, 2009). En el ámbito laboral, el estrés es responsable de un enorme impacto socioeconómico para empleadores, empleados y Estados, incluidos en esta suma los gastos de tratamientos médicos, licencias laborales, jubilaciones por discapacidad y, por supuesto, las caídas en la productividad (Prado, 2016).

La profesión docente y el contexto en que se desempeña reúnen una serie de condiciones que la convierten en una profesión de riesgo (Moraes, Camargo, Welter y Guisso, 2010), hasta el punto que la propia Organización Internacional del Trabajo la consideró ya en 1981 como una profesión de riesgo físico y mental (Prieto, 2011). Los docentes están sometidos a riesgos derivados de las condiciones de seguridad, riesgos higiénicos ocasionados por la exposición a agentes biológicos, químicos y físicos, riesgos ergonómicos y especialmente riesgos psicosociales. Uno de cada tres docentes presenta una o varias de estas patologías (Extremera, Rey y Pena, 2010) que acaban afectando tanto a su salud personal como a la calidad de la enseñanza (Castillo, Fernández y López, 2014).

Es muy numerosa la literatura científica que demuestra que las presiones y el estrés del entorno laboral afectan al estado de salud del personal docente y además pueden impactar negativamente en el propio proceso educativo (Ratto, García-Pérez, Silva y González, 2015). Vergara-Mendoza, Carabajo-Romero y Leal-Maridueña (2017) citan, entre los factores psicosociales que generan problemas de salud en los docentes, la sobrecarga laboral, la baja compensación económica en relación al grado de responsabilidad del cargo, problemas familiares, falta de recreación o un inadecuado clima institucional, entre otros. Entre los riesgos psicosociales más prevalentes, en la literatura científica se refiere el agotamiento de los profesionales con amplias exigencias sociales, que se empezó a abordar intensamente sobre todo en Estados Unidos a partir de la década de los setenta del siglo pasado con el término anglosajón burnout (Bermejo, 2016; Marenco y Ávila, 2016).

El burnout ha sido referido por científicos de todo el mundo (Guan, Xiaerfuding, Ning, Lian, Jiang, Liu y Ng, 2017; Zhenxing y Zhang, 2015; Bozorgi, Laali y Mohammadi-kia, 2014), informándose comúnmente como un resultado del estrés ocupacional crónico (Leiter y Maslach, 2016). Como decimos, poco a poco, el síndrome de burnout se ha ido consolidando como un tema de estudio de gran relevancia, primero en Estados Unidos y posteriormente, en la década de los ochenta en Europa. A mediados los noventa se extiende su estudio a Asia, Oriente Medio, Latinoamérica, Austria y Nueva Zelanda y finalmente, a principios del siglo XXI, también a China, India y África (Schaufeli, Leiter y Maslach, 2009).

Explorar el verdadero alcance del agotamiento se ha vuelto cada vez más importante en las últimas décadas, considerando los altos costos sociales y consecuencias individuales producidas, tales como la depresión, ansiedad, trastornos del sueño, bajo rendimiento laboral, falta de empatía y agresividad (Popa-Velea, Diaconescu, Gheorghe, Olariu, Panaitiu, Cernitanu, Goma y Nivoc, 2019). La literatura apunta, desde hace varias décadas, a mostrar que la docencia es una ocupación de más estrés que la mayoría de otros oficios (Extremera,

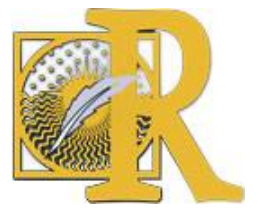

Fecha de recepción: 05-06-2020 Fecha de aceptación: 09-06-2020

Cecilia-Martín, M., Rubio-González, L., Morón-Marchena, J. A. \& Cobos-Sanchiz, D. (2020). Burnout docente:

un análisis bibliométrico sobre la producción científica indexada en Scopus

International Journal of Educational Research and Innovation (IJERI), 14, 197-210

ISSN: 2386-4303 DOI https://doi.org/10.46661/ijeri.4949 
Rey y Pena, 2010). Algunos estudios refieren, de hecho, que los docentes registran niveles de estrés por encima de los médicos, el personal de enfermería (Leonelli, 2013) y la policía (Paredes, 2012).

Los profesionales de la enseñanza constituyen un colectivo tradicionalmente estudiado en sus condiciones laborales y en las repercusiones que ellas tienen sobre los mismos, aunque la investigación sobre estrés laboral en el sector educativo se ha centrado principalmente en educación primaria y secundaria, siendo más escasos los estudios sobre el estrés laboral en el ámbito universitario (Kataoka, Ozawa, Tomotake, Tanioka y King, 2014). No obstante, el ambiente académico universitario también ha sido considerado como un lugar que puede generar estrés al profesorado por factores psicosociales y organizativos del trabajo, como la inseguridad laboral, las propias condiciones de trabajo, la devaluación social de la imagen del profesor, los bajos salarios, la intensidad de la exposición a agentes de riesgo, la falta de recursos humanos y materiales y el agotamiento físico debido al aumento del ritmo y la intensidad de trabajo (Soares, Mafra y De Faria, 2019) y está empezando a generar cada vez mayor interés para los investigadores.

Varios estudios señalan las jornadas extensas, falta de descanso en la jornada y fuera de ella, carga afectiva y emocional, riesgo ante violencia interpersonal, déficit de control sobre el trabajo por falta de recursos materiales, inseguridad, e inestabilidad laboral (UNESCO, 2005) entre otros factores de riesgo. Kuri-Casco (2015) apunta como una de las razones del estrés en este ámbito, a los procesos laboriosos y, hasta cierto punto, burocráticos, de las reformulaciones de proyectos curriculares y modificaciones de planes y programas de estudio. Soares et al (2019) ponen el énfasis en la propia carga docente, el aumento de la participación en tareas administrativas y el nivel de presión que existe sobre la producción científica del profesor-investigador. En concreto, sobre la presión por publicar existe, según Lima y Lima-Filho (2009), una búsqueda casi "frenética" para aumentar la producción que desemboca en cansancio, estrés y, a menudo, frustración.

Por otra parte, en la línea de lo que apunta González-Argote (2019), el crecimiento de la información científica ha sido exponencial en los últimos años y se relaciona, en parte, con el aumento de las revistas científicas y el desarrollo de las TIC. El vasto número de publicaciones científicas, ha hecho aumentar el interés por desarrollar instrumentos que permitan el estudio de las áreas de conocimiento y tópicos de investigación, mediante procedimientos de sistematización y análisis bibliométricos (Pérez, Anta, Badera, García, Pérez y Sarrate, 2003). Este tipo de aproximaciones tienen la utilidad de apoyar el examen y comprensión de los datos históricos, la evolución de las investigaciones e incluso de direccionar su desarrollo futuro (Van Eck y Waltman, 2010).

Dada la actualidad de la temática y el interés investigador creciente sobre Burnout y Burnout en la Enseñanza que venimos describiendo, hemos querido desarrollar un estudio bibliométrico específico para conocer la productividad diacrónica de los veinte últimos años sobre este tópico de investigación. Este trabajo pretende además verificar si se cumple la Ley de Price, determinar qué países son los que producen más documentos científicos sobre el tema abordado, señalar cuáles son las áreas temáticas principales y las revistas dominantes en las que se enmarcan las publicaciones, así como establecer las conexiones entre los distintos descriptores relacionados con las palabras clave analizadas.

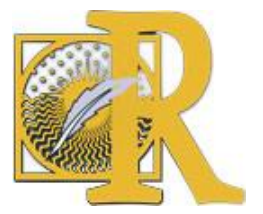

Fecha de recepción: 05-06-2020 Fecha de aceptación: 09-06-2020

Cecilia-Martín, M., Rubio-González, L., Morón-Marchena, J. A. \& Cobos-Sanchiz, D. (2020). Burnout docente:

un análisis bibliométrico sobre la producción científica indexada en Scopus

International Journal of Educational Research and Innovation (IJERI), 14, 197-210

ISSN: 2386-4303 DOI https://doi.org/10.46661/ijeri.4949 


\section{Métodos.}

Se ha realizado un estudio bibliométrico descriptivo, siguiendo la metodología propia de estos estudios (Sampieri y Trejo, 2015). Debido a que el acceso total a la producción científica es inalcanzable, el análisis bibliométrico ha de desarrollarse a través de bases de datos confiables y ampliamente avaladas por la comunidad investigadora. En este sentido, se ha hecho una identificación de artículos científicos indexados en la base de datos Scopus sobre Burnout y Burnout en docentes, en los últimos veinte años (1999-2019).

Las búsquedas se realizaron utilizando operadores booleanos para filtrar los resultados. Los trabajos de búsqueda de los datos y su análisis han tenido lugar durante el mes de marzo de 2020. Se empleó Scopus por ser considerada una de las bases de citas y resúmenes de la literatura con revisión por pares, de amplio espectro temático y más robusta. Las unidades de análisis utilizadas fueron las palabras clave $(\mathrm{KW}+)$, extraídas de forma automática. Con las KW+ obtenidas se crearon mapas bibliométricos aplicando una metodología basada en análisis de co-palabras, técnicas de agrupamiento o clustering y técnicas de visualización. En concreto, para el procesamiento estadístico se empleó Microsoft Office Excel y los resultados se presentaron resumidos en gráficos. Para los mapas bibliométricos se empleó WOSviewer (Van-Eck y Waltman, 2010), un software libre para la representación y análisis de la información, que se presenta como una alternativa a las técnicas tradicionales de representación multidimensional. VOSviewer combina técnicas de visualización y clustering para favorecer el análisis. De los varios tipos de representaciones que ofrece, se optó por utilizar el mapa etiquetado con nombres, donde cada descriptor está representado por una etiqueta y cuyo tamaño es proporcional a su peso. El mapa de redes entre descriptores muestra las relaciones existentes entre las diferentes palabras clave que aparecen en los artículos seleccionados.

\section{Resultados.}

Introduciendo la keyword Burnout en el motor de búsqueda, y filtrando los resultados a publicaciones aparecidas en los últimos veinte años [KEY (burnout) AND PUBYEAR $>1998$ AND PUBYEAR < 2020], obtuvimos un total de 18.047 documentos. En el caso de las KW+ Burnout + Teaching [KEY (burnout AND teaching) AND PUBYEAR > 1998 AND PUBYEAR $<2020$ ], obtuvimos 393 documentos.

\subsection{Productividad diacrónica de los veinte últimos años.}

Para obtener el indicador de productividad, analizando los 18.047 documentos indexados en la base de datos Scopus, que contienen la $\mathrm{KW}+$ Burnout, acotando la búsqueda a los años comprendidos entre 1999 y 2019 y utilizando como variable el año de publicación, podemos observar el aumento en los últimos veinte años de la producción científica que trata sobre el tema. La figura 1 muestra los resultados por año de producción. Se puede observar gráficamente una subida exponencial de la misma que se sigue incrementando en la actualidad, tanto en Burnout como en Burnout en la Enseñanza, siendo el año más productivo justamente el más reciente (2019).

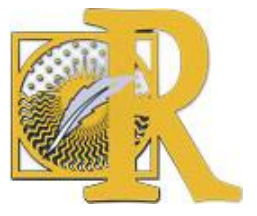

Fecha de recepción: 05-06-2020 Fecha de aceptación: 09-06-2020

Cecilia-Martín, M., Rubio-González, L., Morón-Marchena, J. A. \& Cobos-Sanchiz, D. (2020). Burnout docente:

un análisis bibliométrico sobre la producción científica indexada en Scopus

International Journal of Educational Research and Innovation (IJERI), 14, 197-210

ISSN: 2386-4303 DOI https://doi.org/10.46661/ijeri.4949 


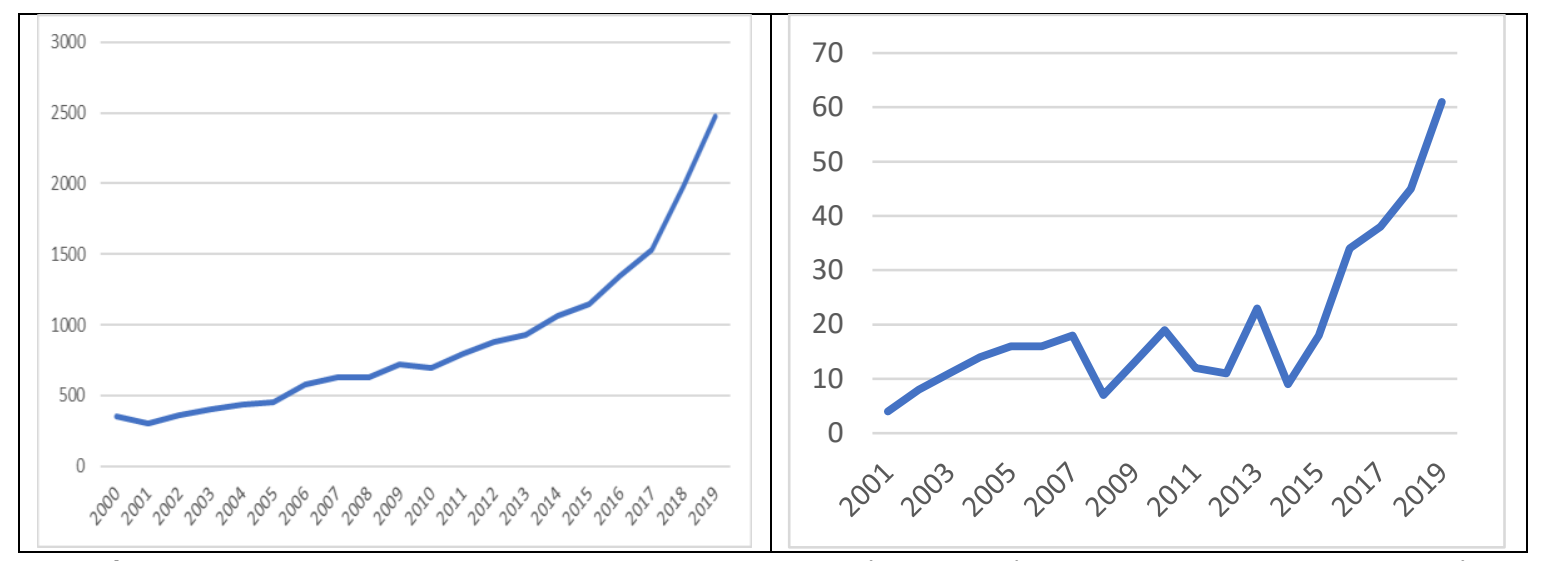

Figura 1. Número de documentos publicados sobre Burnout (izquierda) y sobre Burnout+Teaching (derecha).

\subsection{Liderazgo de producción por países.}

La figura 2 recoge sendos diagramas de Pareto, que muestran la distribución de los datos en orden descendente de frecuencia, con una línea acumulativa en un eje secundario como un porcentaje del total. Se puede observar que un reducido número de países concentran la mayoría de la producción.

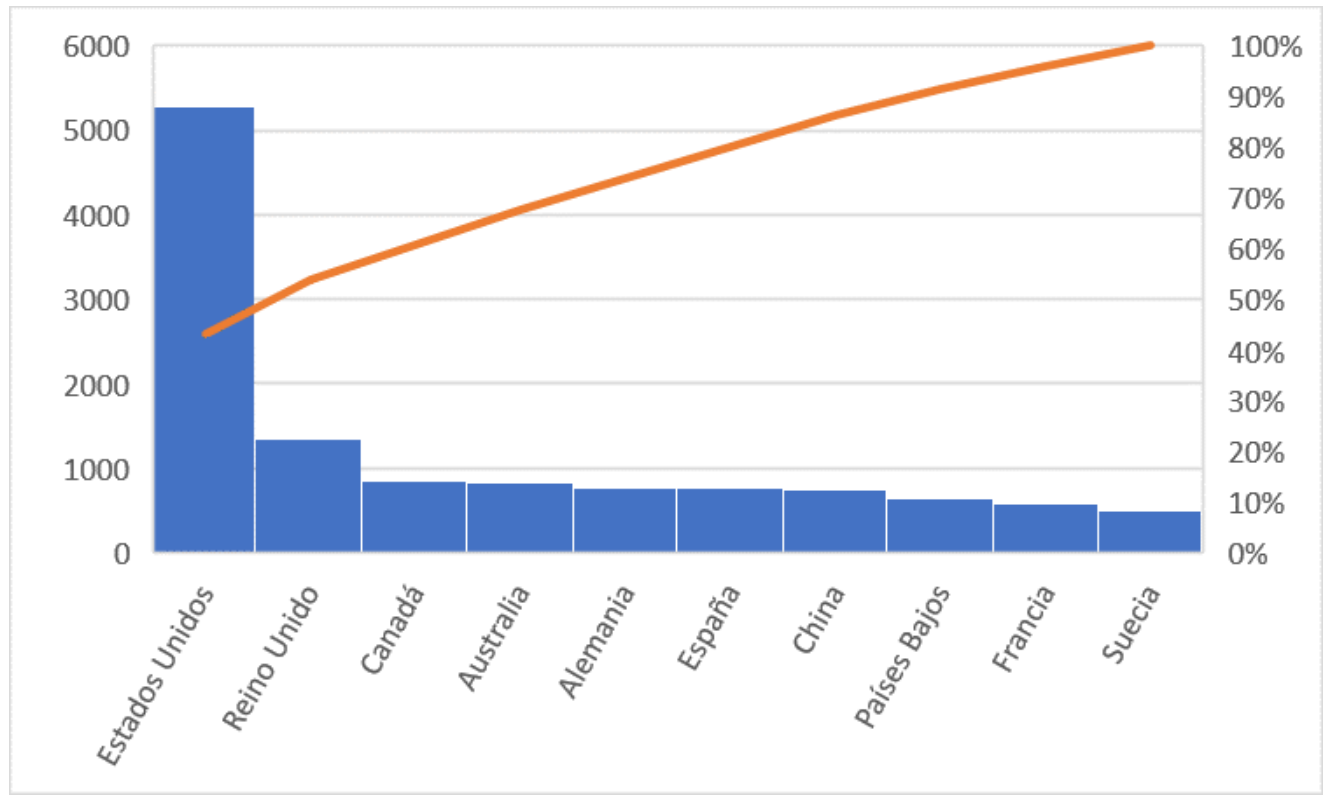

Fecha de recepción: 05-06-2020 Fecha de aceptación: 09-06-2020 


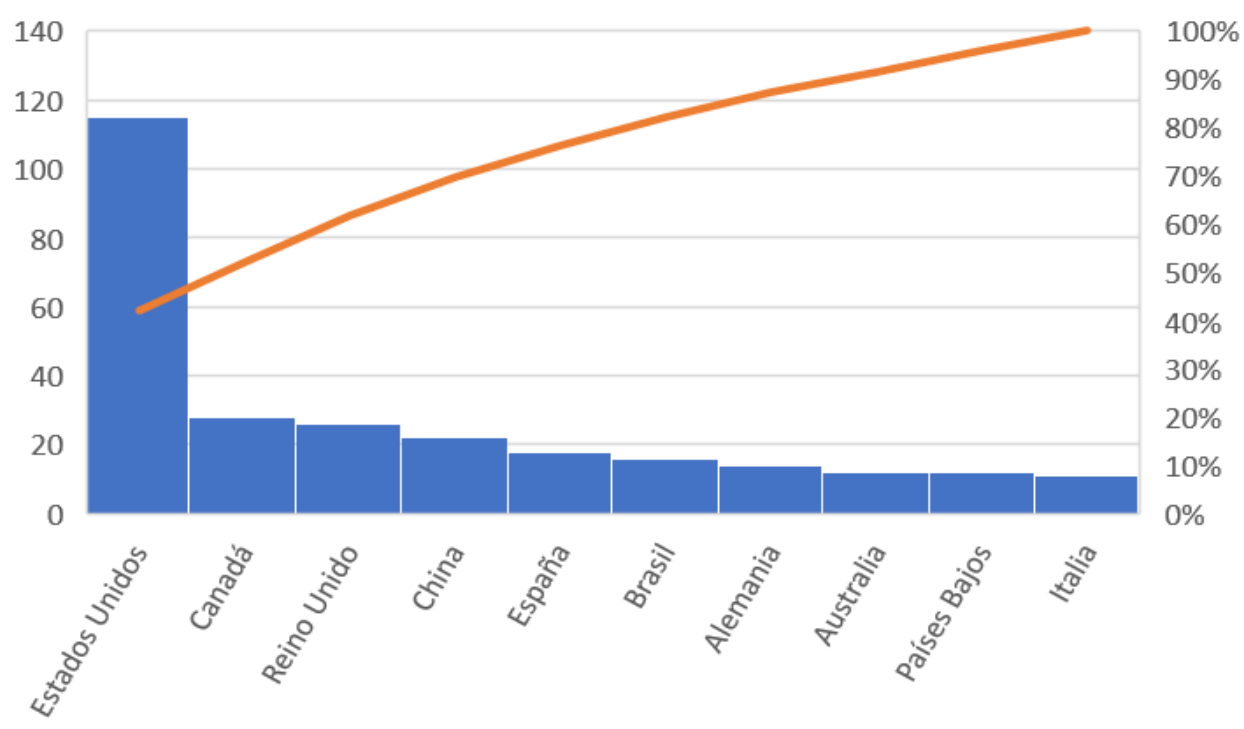

Figura 2. Producción sobre Burnout por países (10 primeros) (cuadro superior) y sobre Burnout + Teaching (cuadro inferior).

En el caso del Burnout, solo Estados Unidos concentra el 29,2\% del total de la producción, mientras que el conjunto de los diez primeros países (Estados Unidos, Reino Unido, Canadá, Australia, Alemania, España, China, Países Bajos, Francia y Suecia, por este orden) ha publicado el $67,9 \%$.

En el caso de Burnout + Teaching los diez primeros países han acaparado el 69,3\% de la producción, siendo Estados Unidos el más productivo con un $29 \%$ del total. Es interesante observar que, si bien, ocho de los diez países repiten en ambos gráficos, Francia y Suecia dejan de estar entre los diez países más productivos cuando se trata de Burnout + Teaching, entrando a ocupar su lugar Brasil e Italia.

\subsection{Principales áreas temáticas y revistas dominantes.}

La figura 3 representa el porcentaje de publicaciones de las diez primeras áreas temáticas. Medicina, Enfermería, Psicología y Ciencias Sociales son, con mucha diferencia, las áreas más representativas, representando el $85 \%$ del total de la producción sobre Burnout y el $84 \%$ en el caso de las publicaciones sobre Burnout + Teaching. 


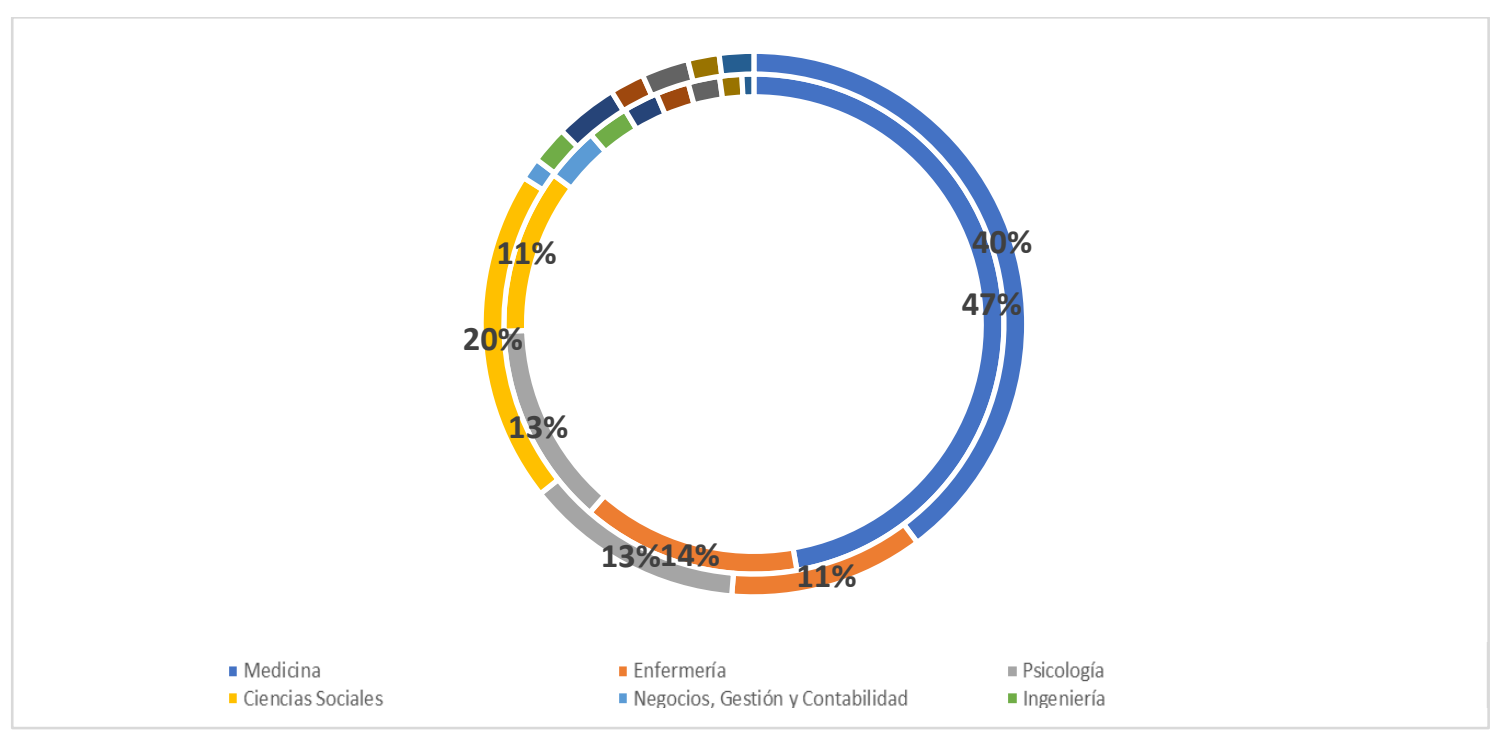

Figura. 3. Porcentaje de publicaciones por áreas temáticas.

En cuanto a las revistas existen, en el caso del Burnout, una serie de publicaciones dominantes que acaparan buena parte de la producción. En concreto, las diez revistas que aparecen en la figura 4 han publicado 1.153 artículos sobre la temática.

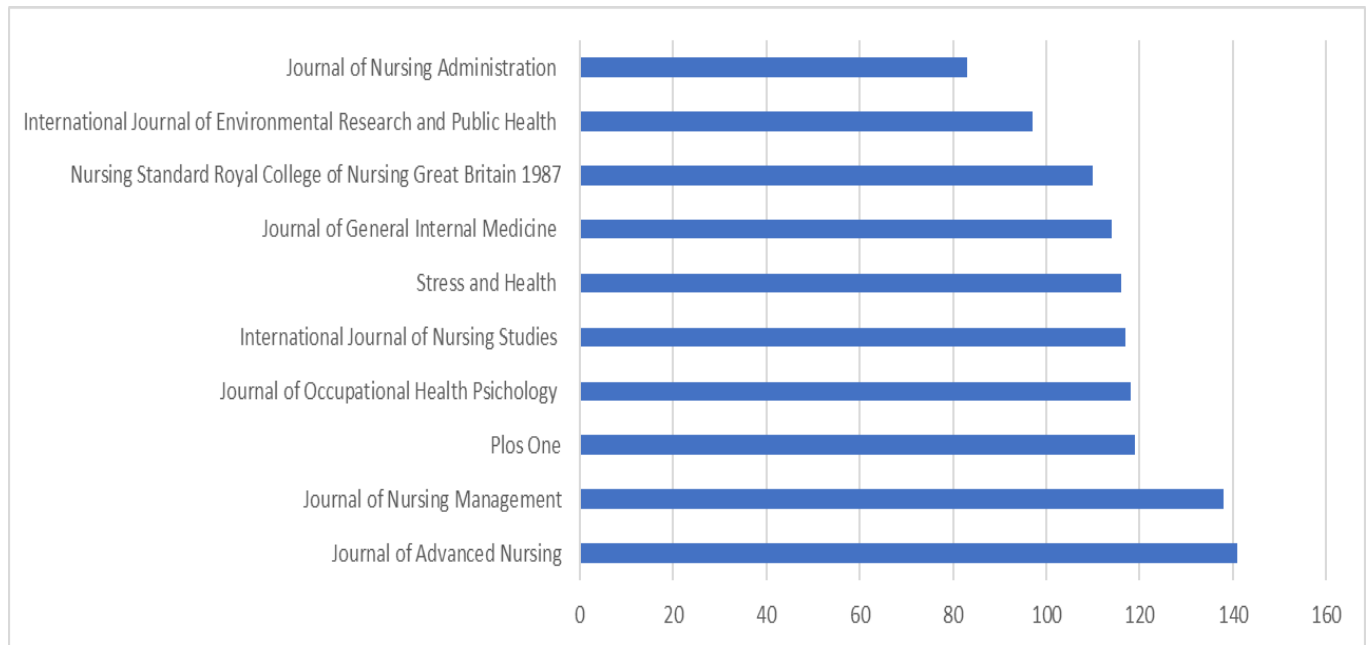

Figura 4. Las revistas dominantes en publicaciones sobre Burnout.

\subsection{Conexiones entre los descriptores relacionados con el Burnout y la Enseñanza.}

El mapa de redes entre descriptores, representado en la figura 5 , muestra las relaciones de co-ocurrencia de palabras clave que existen entre las diferentes palabras que utilizan los 393 artículos analizados que se encuentran indexados en Scopus y contienen las KW+ Burnout + Teaching. Se determinó un número mínimo de 10 ocurrencias, lo que dio lugar a 185 descriptores.

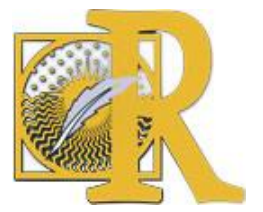

Fecha de recepción: 05-06-2020 Fecha de aceptación: 09-06-2020 


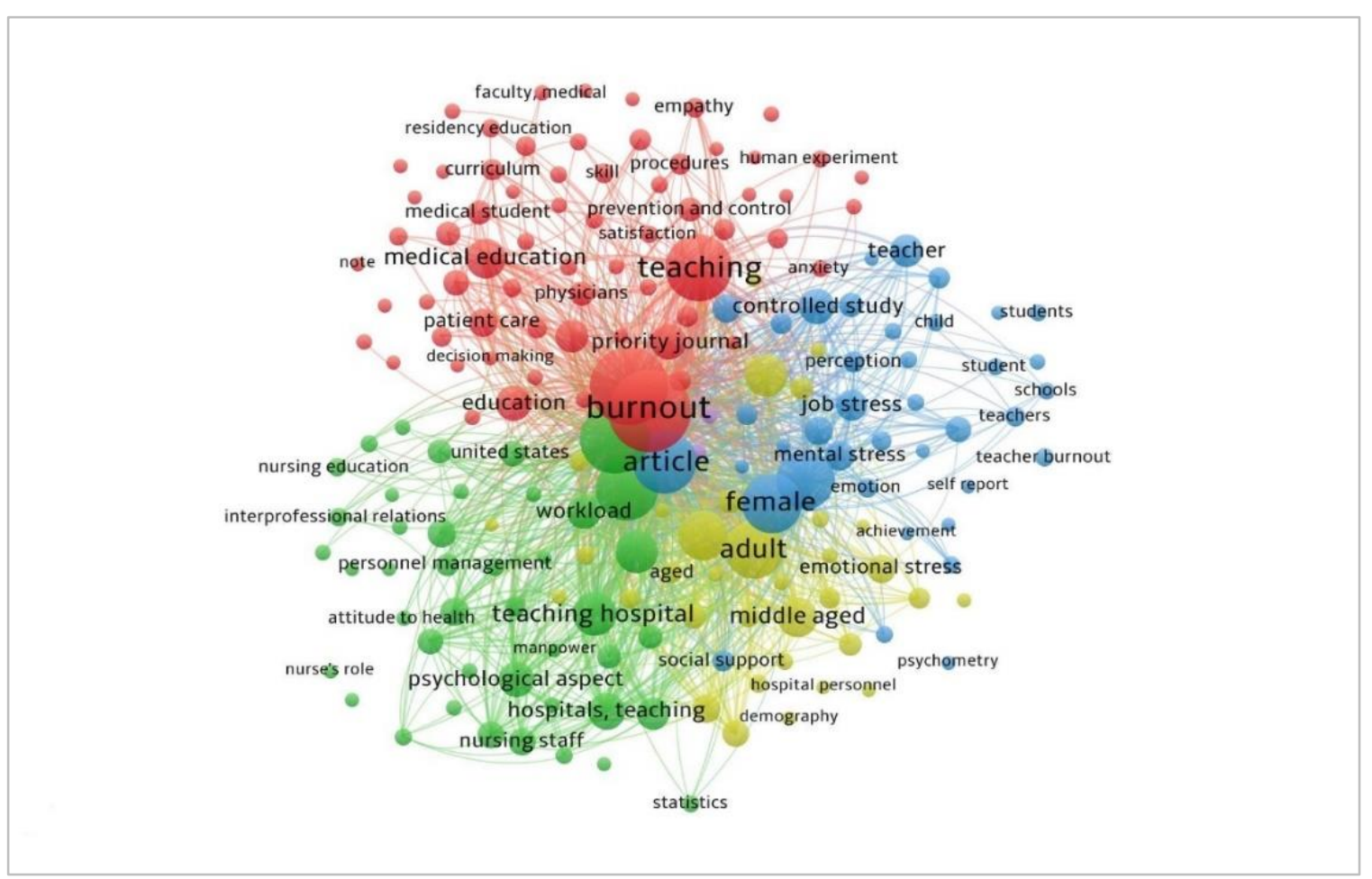

Figura 5. Mapa de redes entre las palabras clave de los artículos seleccionados.

Observando la imagen, se pueden distinguir hasta cinco grupos de descriptores diferenciados por distintos colores, que poseen un gran entramado entre sí, siendo el tamaño de los conceptos proporcional a la frecuencia de aparición y al número de conexiones con otros descriptores.

Cuatro de ellos destacan por tener mayor densidad y concentrar un número significativo de relaciones entre los distintos ítems.

El grupo de color rojo está muy relacionado con la educación médica: curriculum, estudiantes de medicina, competencias profesionales, facultades, escuelas de medicina, etc.

Por su parte el clúster de color verde hace referencia, sobre todo, a burnout profesional y satisfacción en el trabajo específicamente en el sector de la enfermería.

Los descriptores en azul relacionan el burnout con la enseñanza en términos generales (escuela, maestros, estudiantes, universidad...)

El grupo amarillo, por su parte, gira en torno a factores sociodemográficos como la edad (niños, adolescentes, jóvenes, adultos...) o el estado civil.

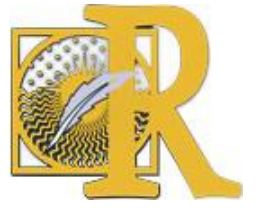




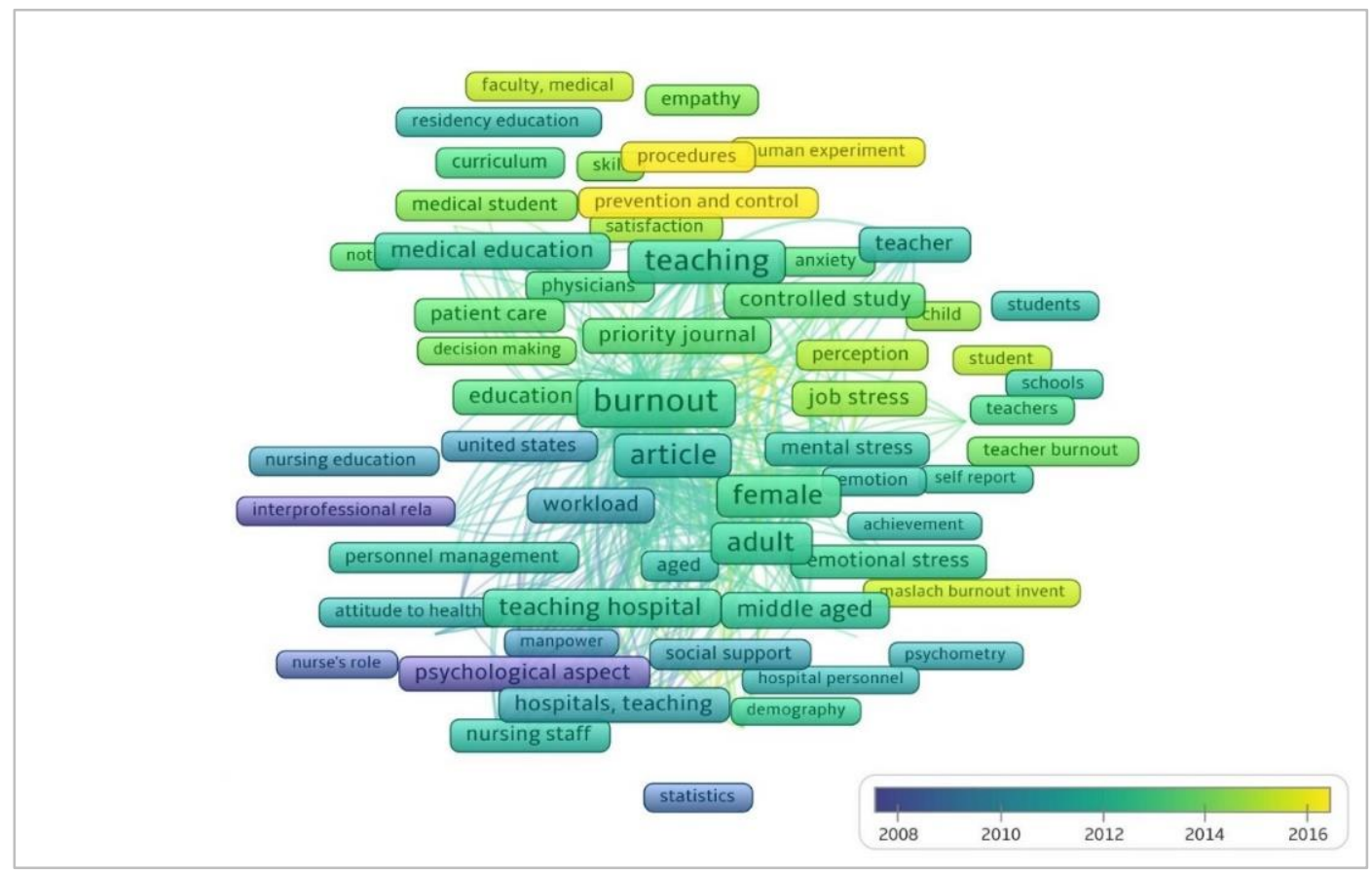

Figura 6. Mapa temporal de palabras clave.

Finalmente, la figura 6 muestra un mapa temporal donde se pueden apreciar las palabras más antiguas y los términos emergentes, destacando entre estos últimos los propios procesos, herramientas, instrumentos y metodologías de investigación: inventario de burnout de Maslach, procedimientos, experimentos con humanos, etc.

\section{Discusión.}

Atendiendo a la ley de crecimiento exponencial de Price, se puede comprobar que se cumple la premisa que afirma que la producción científica se duplica cada 10-15 años (Price, 1963). En este caso, la proporción es incluso mayor. De 344 documentos publicados en 1999, se pasa a 720 en 2009, 1.149 en 2015 y 2.479 en 2019.

En el caso de la combinación Burnout + Teaching, aunque la distribución de la producción es más irregular, igualmente se cumple la ley: se publican 9 documentos en 1999, 18 en 2015 y 61 en 2019. Se ha constatado que, en ambos casos, estamos en una fase de crecimiento exponencial que aparentemente aún no se ha agotado. En otras palabras, en estos momentos esta temática es un frente de investigación de plena actualidad.

Si consultamos la literatura especializada sobre un tema determinado, encontramos que la mayor parte de trabajos sobre él se encuentran publicados en un pequeño número de revistas denominado núcleo, en la línea de lo que apuntaba Bradford (Ardanuy, 2012).

En este caso, las revistas más importantes en cuanto a Burnout en, en términos de cantidad de publicaciones son Journal of Advanced Nursing (141 general artículos), y Journal of Nursing Management (138), seguidas por un grupo de seis revistas que publican más de 100 artículos cada una de ellas.

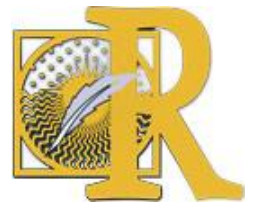


Sin embargo, en el caso de la producción sobre Burnout + Teaching no hay revistas que dominen de esta forma tan clara existiendo, de hecho, una enorme dispersión. Hasta 25 revistas publican 4 ó 3 artículos sobre el tema, siendo 4 el número máximo de artículos publicados por cada una de ellas.

Tal vez, lo más interesante sea constatar que la preocupación de los investigadores por el Burnout trasciende a las Ciencias Médicas, convirtiéndose en una preocupación interdisciplinar de varios campos temáticos que incluyen también a la Psicología y las Ciencias Sociales en general.

Sobre la producción por países existe también una alta concentración, en este caso geográfica, totalmente aglutinada por un conjunto de diez países que, solo en el caso de Burnout, publican 12.261 sobre 18.047 artículos, destacando claramente entre ellos los Estados Unidos, Reino Unido, Canadá y Australia.

Price constató que la literatura científica pierde actualidad cada vez más rápidamente, lo que denominó Ley de obsolescencia de la bibliografía, aunque también señaló que dicho envejecimiento no es uniforme para todas las disciplinas científicas, siendo mayor en las ciencias experimentales y tecnologías que en las humanidades (Ardanuy, 2012).

En el caso que nos ocupa, efectivamente se aprecia una evolución clara en los intereses de investigación pasándose de un interés inicial en los campos de la enfermería y la medicina, que pasa después por la enseñanza para finalmente hacer un mayor hincapié por parte de los investigadores en los propios procesos, herramientas, instrumentos y metodologías de investigación.

Finalmente, podemos concluir, en la línea de lo que apunta Gálvez (2016) que, debido al carácter intersectorial, multidisciplinar y multidimensional de los tópicos de conocimiento analizados, los mapas bibliométricos mejoran la comprensión global de la estructura semántica y conceptual de este campo de investigación y ayudan a comprender su estructura temática.

\section{Limitaciones del estudio.}

Aunque Scopus es una de las bases de datos de literatura científica más robustas y completas, la mayor limitación de este estudio es haberse circunscrito solo a la misma. Como futuras líneas de investigación, se puede establecer un análisis comparativo con otras bases de datos, tales como Science Citation Index, Social Science Citation Index, Scielo y/o Pubmed, para así ampliar y tener una visión más general de las líneas de investigación en el conjunto de bases de datos más relevantes a nivel internacional.

\section{Referencias bibliográficas.}

- Ardanuy, J. (2012). Breve introducción a la Bibliometría. Barcelona: Universitat de Barcelona.

- Bermejo, L. (2016). Bienestar docente. Estrategias para una vida emocionalmente más saludable en el trabajo del profesor. Revista Padres y Maestros, 368, 18-23. Recuperado de: https://revistas.comillas.edu/index.php/padresymaestros/article/view/7517

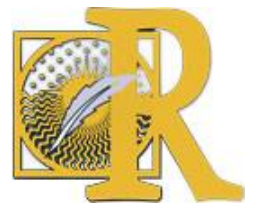

Fecha de recepción: 05-06-2020 Fecha de aceptación: 09-06-2020

Cecilia-Martín, M., Rubio-González, L., Morón-Marchena, J. A. \& Cobos-Sanchiz, D. (2020). Burnout docente:

un análisis bibliométrico sobre la producción científica indexada en Scopus

International Journal of Educational Research and Innovation (IJERI), 14, 197-210

ISSN: 2386-4303 DOI https://doi.org/10.46661/ijeri.4949 
- Bozorgi, F., Laali, A. y Mohammadi kia, A. (2014). Prevalence and factors associated with burnout in emergency medical workers in Mazandaran University of Medical Sciences. Journal of Mazandaran University of Medical Sciences, 24 (112), 2-7. Recuperado de: http://jmums.mazums.ac.ir/article-1-3306-en.html

- Castillo, A., Fernández, R. y López, P. (2014). Prevalencia de ansiedad y depresión en docentes. Enfermería del Trabajo, 4, 55-62. Recuperado de: https://dialnet.unirioja.es/servlet/articulo?codigo $=4738761$

- European Agency for Safety and Health at Work (2007). Expert forecast on emerging psychosocial risks related to occupational safety and health. Luxemburgo: Office for Official Publications of the European Communities.

- Extremera, N., Rey, L. y Pena, M. (2010). La docencia perjudica seriamente la salud. Boletín de Psicología, 100, 43-54. Recuperado de: http://www.uv.es/seoane/boletin/previos/N100-3.pdf

- Gálvez, C. (2016). Visualización de las principales líneas de investigación en salud pública: un análisis basado en mapas bibliométricos aplicados a la Revista Española de Salud Pública (2006-2015). Revista Española de Salud Pública, 90e1-e10. Recuperado de: https://dialnet.unirioja.es/servlet/autor?codigo $=4854914$

- Gil-Monte, P. (Coord.) (2014). Manual de psicosociología aplicada al trabajo y a la prevención de riesgos laborales. Madrid: Pirámide.

- González-Argote, J. (2019). La producción científica latinoamericana sobre historia clínica digital: un análisis desde Scopus. Revista Cubana de Salud Pública, 45 (3), e1312.28. Recuperado de: http://www.revsaludpublica.sld.cu/index.php/spu/article/view/1312/1386

- Guan, S., Xiaerfuding, X., Ning, L., Lian, Y., Jiang, Y., Liu, J. y Ng, T.B. (2017). Effect of job strain on job burnout, mental fatigue and chronic diseases among civil servants in the Xinjiang Uygur Autonomous Region of China. International Journal of Environmental Research and Public Health, 14 (8), 872. Recuperado de: https://www.mdpi.com/16604601/14/8/872

- Kataoka, M., Ozawa, K., Tomotake, M, Tanioka, T. y King, B. (2014). Occupational stress and its related factors among university teachers in Japan. Healh, 6 (5), 299-305. Recuperado de: https://m.scirp.org/papers/43101

- Kuri-Casco, S.A. (2015). El estrés. ¿promueve o dificulta la innovación? IJERI: International Journal of Educational Research and Innovation, 9, 1-11. Recuperado de: https://www.upo.es/revistas/index.php/lJERI/article/view/1453

- Leiter M.P. y Maslach, C. (2016). Latent burnout profiles: A new approach to understanding the burnout experience. Burnout Research, 3 (4), 89-100. Recuperado de: https://www.sciencedirect.com/science/article/pii/S2213058615300188

- Leonelli, L.B. (2013). Estresse percebido em profissionais da atenção primária à Saúde [tesis]. São Paulo: Universidade Federal de São Paulo. 
- Lima, M. de F.E.M. y Lima-Filho, D. de O. (2009). Condições de trabalho e saúde do/a professor/a universitário/a. Ciências \& Cognição, 14 (3), 62-82. Recuperado de:

http://pepsic.bvsalud.org/scielo.php?script=sci abstract\&pid=S1806-

$58212009000300006 \&$ lng $=$ pt\&nrm=iso

- Marenco, A.D. y Ávila, J.H. (2016). Burnout y problemas de salud mental en docentes: diferencias según características demográficas y sociolaborales. Psychología: Avances de la Disciplina, 10 (1), 91-100. Recuperado de: https://dialnet.unirioja.es/servlet/articulo?codigo $=5735299$

- Moraes, R., Camargo, J., Welter, M.M. y Guisso, L. (2010). Saúde docente, condições e carga de trabalho. Revista Electrónica de Investigación y Docencia, 4, 147-160. Recuperado de: https://revistaselectronicas.ujaen.es/index.php/reid/article/view/1024

- Paredes, D.S. (2012). Nível de Atividade Física e Nível de Estresse de Policiais Militares do $16^{\circ}$ BPM de Santa Catarina. Florianópolis: Universidade Federal de Santa Catarina.

- Patlán, J. (2019). ¿Qué es el estrés laboral y cómo medirlo? Salud Uninorte, 35 (1), 1-29. Recuperado de: http://rcientificas.uninorte.edu.co/index.php/salud/article/viewArticle/8738

- Pérez, G., Anta, C., Badera, S., García, J., Pérez, M. y Sarrate, M. (2003). Análisis bibliométrico en Educación. Incidencia en la calidad universitaria. Madrid: Ministerio de Educación y Ciencia de España.

- Popa-Velea, O., Diaconescu, L.V., Gheorghe, I.R., Olariu, O., Panaitiu, I., Cerniţanu, M., Goma, L. y Nicov, I. (2019). Factors associated with burnout in medical academia: An exploratory analysis of romanian and moldavian physicians. International Journal of Environmental Research and Public Health, 16(13). Recuperado de: https://europepmc.org/article/PMC/6650893

- Prado, C.E.P. (2016). Estresse ocupacional: causas e consequências. Revista Brasileira de Medicina do Trabalho, 14 (3), 285-289. Recuperado de: http://www.rbmt.org.br/details/122/pt-BR/estresse-ocupacional--causas-e-consequencias

- Price, D.J.S. (1963). Little science, big science. New York: Columbia University Press.

- Prieto, M. (2011). Estrés y ansiedad en profesores. Crítica, 974, 42-45. Recuperado de: https://dialnet.unirioja.es/servlet/articulo?codigo=3694330

- Ratto, A., García-Pérez, R.C., Silva, M.I. y González, M.C. (2015). El síndrome de quemarse por el trabajo y factores psicosociales en docentes de primaria de la ciudad de Montevideo. Ciencias Psicológicas, 9 (2), 273-281. Recuperado de:

http://www.scielo.edu.uy/scielo.php?script=sci arttext\&pid=S1688-42212015000300005

- Sampieri, R. y Trejo, M.A. (2015). Mapas bibliométricos como herramienta en la organización y análisis en Ciencia. Revista de Educación Bioquímica, 34 (4), 93-97. Recuperado de:

https://www.researchgate.net/publication/325261254 MAPAS BIBLIOMETRICOS COMO HER RAMIENTA EN LA ORGANIZACION Y ANALISIS EN CIENCIA 
- Schaufeli, W., Leiter, M. y Maslach, C. (2009). Burnout: 35 years of research and practice. Career Development International, 14 (3), 204-220. Recuperado de: https://www.wilmarschaufeli.nl/publications/Schaufeli/311.pdf

- Soares, M.B., Mafra, S.C.T. y De Faria, E.R. (2019). Factors associated with perceived stress among professors at a federal public university. Revista Brasileira de Medicina do Trabalho, 17 (1), 90-98. Recuperado de: http://www.rbmt.org.br/details/425/en-US/factorsassociated-with-perceived-stress-among-professors-at-a-federal-public-university

- UNESCO (2005). Condiciones de Trabajo y Salud en el trabajo Docente. Santiago de Chile: Oficina Regional de Educación de la UNESCO. Recuperado de: https://unesdoc.unesco.org/ark:/48223/pf0000144745

- Vallejo, M., Aja, J. y Plaza, J.J. (2018). Estrés percibido en estudiantes universitarios. Influencia del burnout y del engagement académico. IJERI: International Journal of Educational Research and Innovation, 9, 220-236. Recuperado de: https://www.upo.es/revistas/index.php/lJERl/article/view/2558

- Van-Eck, N. y Waltman, L. (2010). Software survey: VOSviewer, a computer program for bibliometric mapping. Scientometrics. 84. 523-538. Recuperado de: https://link.springer.com/article/10.1007/s11192-009-0146-3

- Vergara-Mendoza, K.Z., Carabajo-Romero, I.R. y Leal-Maridueña, I.A. (2017). El nivel de stress en los docentes de la UNEMI. Dominio de las Ciencias, 3 (2), 405-425. Recuperado de:

https://dialnet.unirioja.es/servlet/articulo?codigo $=5889723$

- Zhenxing, G. y Zhang, J. (2015). Job burnout and psychological empowerment among Police in Mainland China. Iranian Journal of Public Health, 44 (7), 1014-1015. Recuperado de: http://ijph.tums.ac.ir/index.php/ijph/article/view/3339 